\title{
ON THE REALIZATION OF CURRENT-MODE INTEGRATOR USING CURRENT CONVEYORS
}

\author{
MUHAMMAD TAHER ABUELMA'ATTI \\ King Fahd University of Petroleum and Minerals, Box 203, Dhahran 31261, Saudi Arabia
}

(Received March 2, 1994; in final form April 13, 1994)

An improved analysis of a recently proposed current-mode integrator [1] is presented. The analysis includes the parasitic serial resistance on port $\mathrm{X}$ of the current conveyor. The feasibility of obtaining an error cancellation is considered.

\section{INTRODUCTION}

In a recent publication, Nandi and Ray [1] present a current-mode integrator/ differentiator using current conveyors. Fig. 1 shows the proposed circuit, which comprises two second-generation current conveyors (CCII). If the CCIIs are assumed to be ideal then the output current can be expressed as

$I_{o}=\left(I_{2}-\lambda I_{1}\right) Z_{1} / Z_{2}$

where $\lambda=r_{2} / r_{1}$. A true differential input capability can be obtained if $r_{2} / r_{1}=1$.

Current conveyors are not ideal. Therefore, errors in the output current $I_{o}$ are expected. Analysis with non-ideal CCIIs having finite current/voltage $\left(\delta_{i, v}\right)$ tracking errors [1] indicates deviations from true differential input capability and modifications to the nominal value of the time constant. However, the tracking errors are not the only non-idealities of the CCIIs. The parasitic serial resistor $r_{X Y}$ on port $\mathrm{X}(\cong 50 \mathrm{Ohm}$ for the AD844 current conveyor) must be taken into consideration.

It is the purpose of this paper to present an improved analysis for the effect of current-conveyor non-idealities, taking into consideration the effect of the parasitic serial resistance $r_{X Y}$ on port X. In contrast with [1], it will be shown that ideal integration and differentiation can be attained, without change in time constant and without recourse to compensating schemes.

\section{ANALYSIS}

The effect of the non-ideal current conveyor characteristic on the performance of the circuit of Fig. 1 can be studied using the simplified model shown in Fig. 2 for the CCII. This simplified model was deduced from reference [2]. Due to the high 


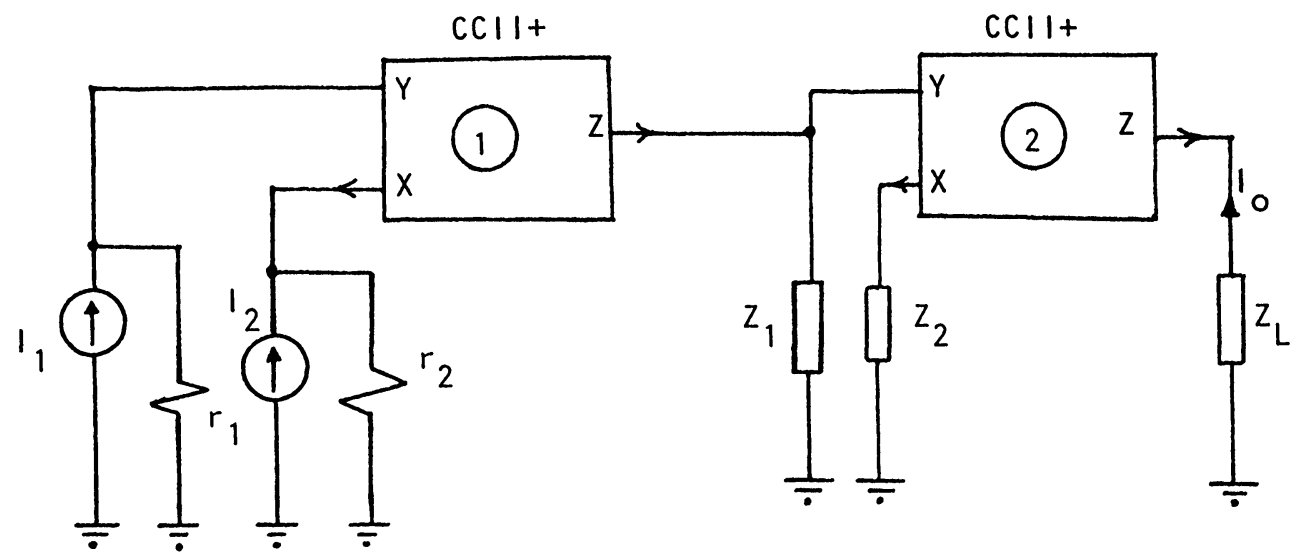

FIGURE 1 Proposed current mode integrator circuit [1]

values of $R_{Y}$ and $R_{Z}$ and the low values of $C_{Y}$ and $C_{Z}$, the effects which have to be considered are principally due to $\delta_{i}, \delta_{\nu}$, and $r_{X Y}$. Assuming that the current transfer ratio and the voltage gain of the non-ideal CCIIs can be described by $\delta_{i k}$ $=i_{Z} / i_{X}, \delta_{\nu k}=\nu_{X} / \nu_{Y}, k=1,2$ where $\delta_{i k}=1+\varepsilon_{i k},\left|\varepsilon_{i k}\right| \ll 1$ and $\delta_{\nu k}=1+\varepsilon_{\nu k}$, $\left|\varepsilon_{v k}\right| \ll 1$, reanalysis of the network of Fig. 1 yields the modified output current $\left(I_{o}^{\sim}\right)$

$I_{o}^{\sim}=\frac{\delta_{i 1} \delta_{i 2} \delta_{\nu 2}\left(I_{2}-\delta_{\nu 1} \frac{r_{1}}{r_{2}} I_{1}\right) Z_{1} / Z_{2}}{\left(1+r_{X X 2} / Z_{2}\right)\left(1+r_{X Y 1} / r_{2}\right)}$

From (2), it is obvious that the output current will be affected by the CCII nonidealities. A first-order correction can be attained, however, by adjusting $r_{2}$ so that
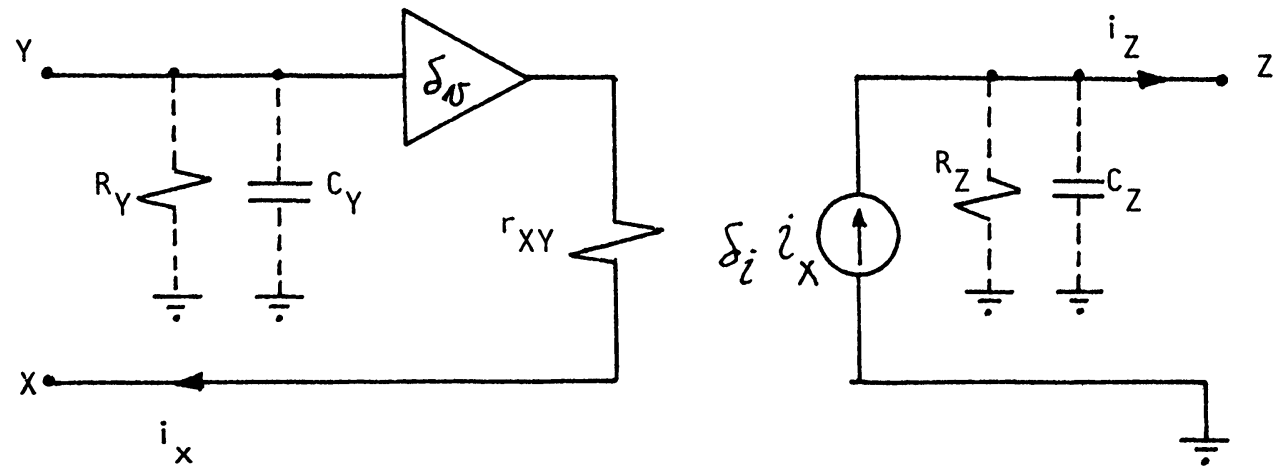

FIGURE 2 Simplified model for nonideal CCII + 
$r_{2}=r_{1} \delta_{\nu 1}$. Thus, (2) reduces to

$I_{o}^{\sim}=\frac{\delta_{i 1} \delta_{i 2} \delta_{\nu 2}\left(I_{2}-I_{1}\right) Z_{1} / Z_{2}}{\left(1+r_{X Y 2} / Z_{2}\right)\left(1+r_{X Y 1} / r_{2}\right)}$

Since the parasitic serial resistance $r_{X Y k}, k=1,2$ is of the order of $10=100 \mathrm{ohms}$ [3], and if we choose $Z_{2} \gg R_{X Y 2}$ and $r_{2} \gg r_{X Y 1}$, then, after neglecting second-order terms, (3) reduces to

$I_{o}^{\sim}=\left(1+\varepsilon_{i 1}+\varepsilon_{i 2}+\varepsilon_{\nu 1}-\frac{r_{X Y 2}}{Z_{2}}-\frac{r_{X Y 1}}{r_{2}}\right) \frac{Z_{1}}{Z_{2}}\left(I_{2}-I_{1}\right)$

Combining (1) (with $r_{2}=r_{1}$ ) and (4), the nominal output current can be expressed as

$\frac{I_{o}^{\sim}}{I_{o}}=1+\varepsilon_{i 1}+\varepsilon_{i 2}+\varepsilon_{\nu 2}-r_{X Y 2} / Z_{2}-r_{X Y 1} / r_{2}$

From (5), it is obvious that the nominal output current is altered owing to the CCII non-idealities. However, (5) contains positive and negative terms and, thus, cancellation of the effects of CCII non-idealities is feasible. This indicates that a proper design can yield an output current that is practically insensitive to the CCII nonidealities. It is worth mentioning that with CCII non-idealities, an ideal integration function is still realizable but with a modified time constant given by

$\tau^{\sim}=C R /\left(1+\varepsilon_{i 1}+\varepsilon_{i 2}+\varepsilon_{\nu 1}-\frac{r_{X Y 2}}{R}-\frac{r_{X Y 1}}{r_{2}}\right)$

where $Z_{1}=1 / s C$ and $Z_{2}=R$. From (6), it is obvious that proper selection of $r_{2}$ and $R$ may result in cancellation of error terms. Thus the integrator time constant, with CCII nonidealities, remains unchanged without using a compensating resistor as suggested in [1].

\section{CONCLUSION}

In this paper an improved analysis for the current-mode integrator circuit of Fig. 1 has been presented. The analysis includes the effect of the parasitic resistance at port $\mathrm{X}$ of the current conveyor as well as the current and voltage tracking errors. The analysis shows that ideal integrators can be realized by careful selection of the active and passive components without recourse to using additional compensating resistor as proposed in [1]. 


\section{REFERENCES}

1. R. Nandi and S.B. Ray, Precise realisation of current mode integrator using current conveyor, Electronics Letters, Vol. 29, 1993, pp. 1152-1153

2. B. Wilson, Performance analysis of current conveyors, Electronics Letters, Vol. 25, 1989, pp. 15961598

3. B. Wilson, Current mode circuits: Analysis and CAD modelling, International Symposium on Circuits and Systems, Vol. 4, 1990, pp. 3242-3245 

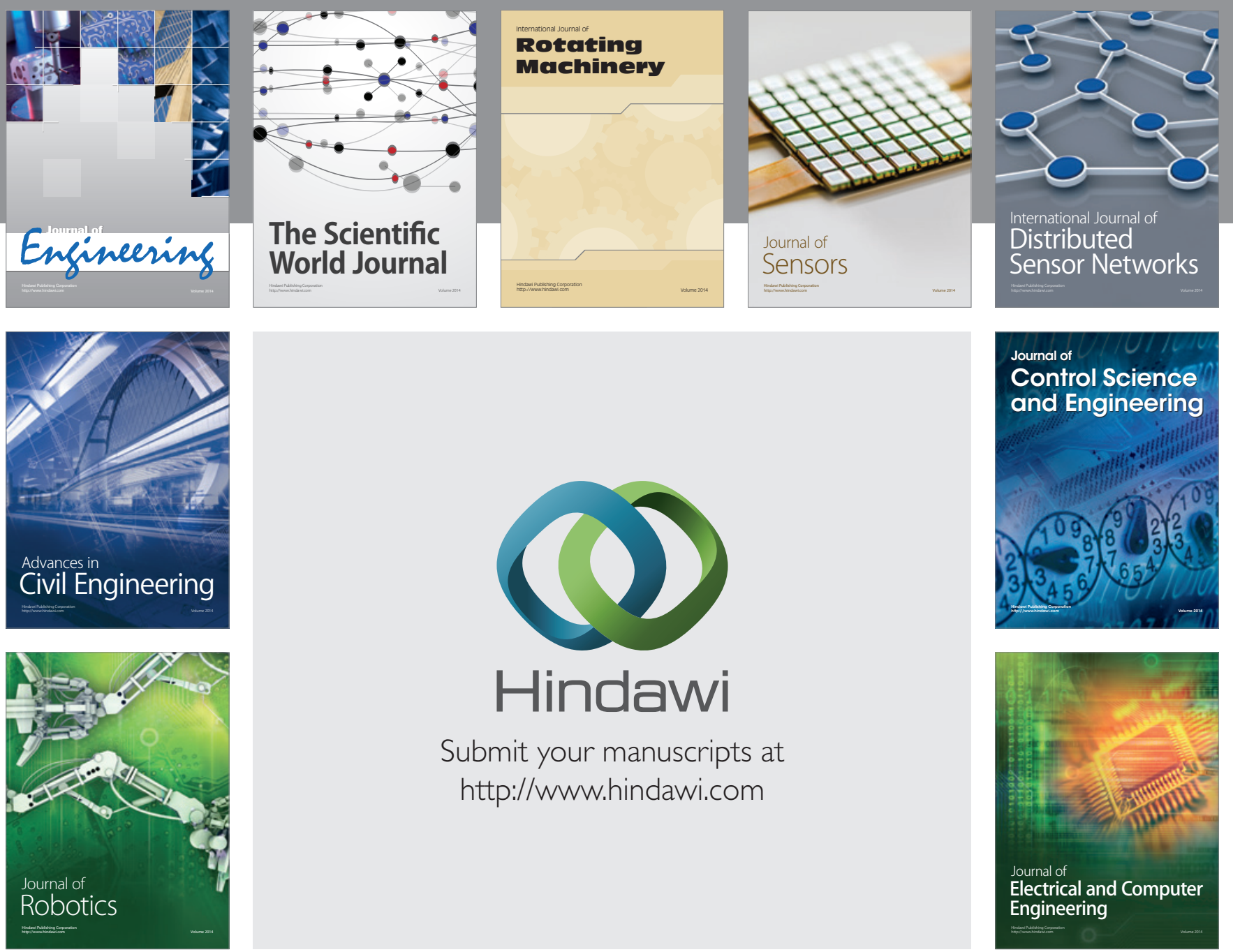

Submit your manuscripts at

http://www.hindawi.com
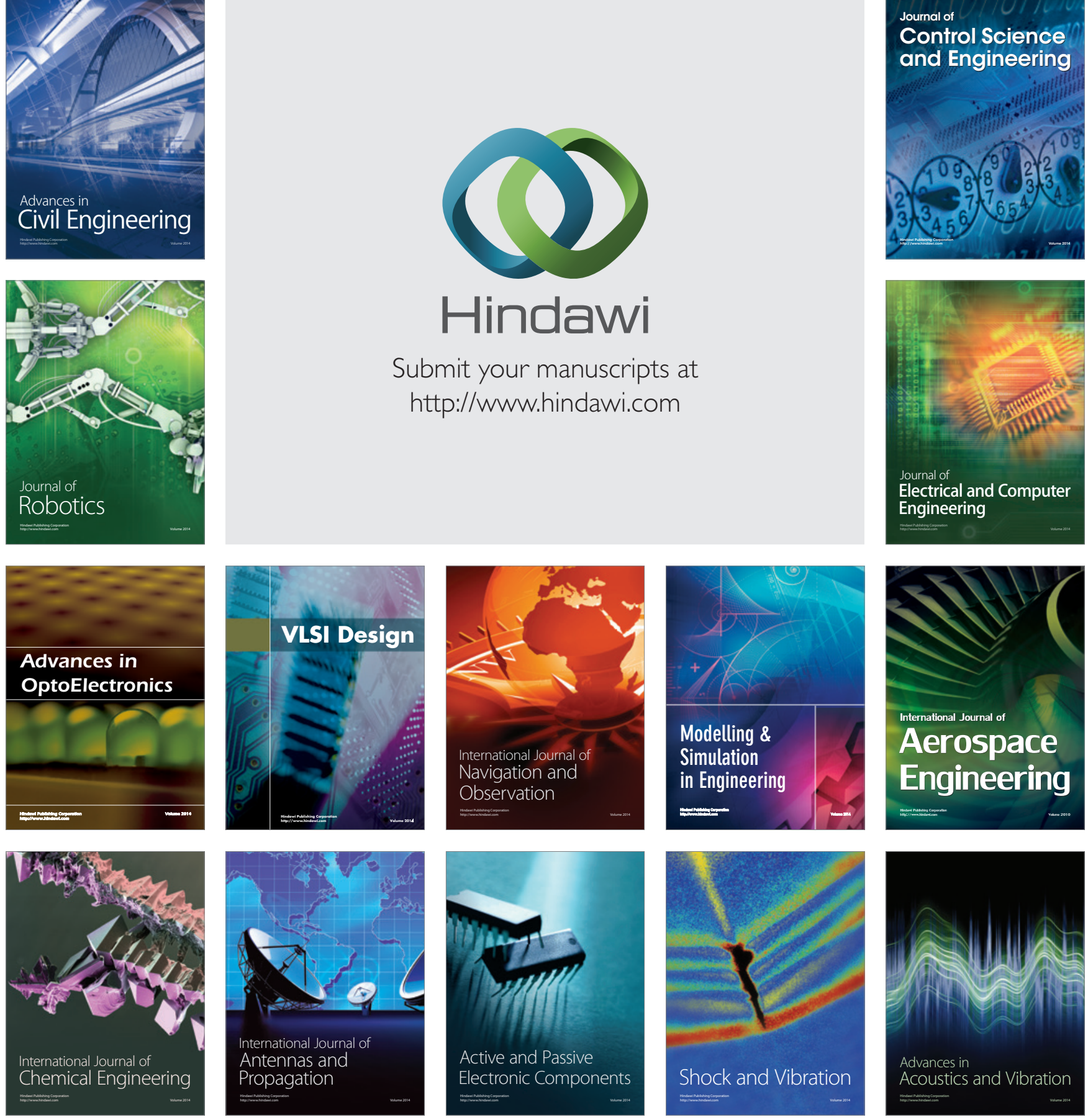